\title{
Transport and Turnover of Microtubules in Frog Neurons Depend on the Pattern of Axonal Growth
}

\author{
Sunghoe Chang, ${ }^{1}$ Vladimir I. Rodionov, ${ }^{2}$ Gary G. Borisy, ${ }^{2}$ and Sergey V. Popov ${ }^{1}$ \\ ${ }^{1}$ Department of Physiology and Biophysics, University of Illinois at Chicago, Chicago, Illinois 60612, and 2Laboratory of \\ Molecular Biology, University of Wisconsin, Madison, Wisconsin 53076
}

\begin{abstract}
The transport of axonal microtubules in growing neurites has been a controversial issue because of clear but conflicting results obtained with fluorescence-marking techniques. We have attempted to resolve the discordance via analysis of the relationship between apparent microtubule translocation and cell adhesion. Neuronal cultures were prepared from Xenopus embryos $1 \mathrm{~d}$ after injection of Cy3-conjugated tubulin into one of the blastomeres of two-cell-stage embryos. Anterograde translocation of axonal microtubules was observed in neurons cultured on a laminin-coated surface, in agreement with previously published data for Xenopus embryonic neurons. How-
\end{abstract}

ever, when neuronal cultures were prepared on a concanavalin A-treated surface, the axonal microtubules were stationary, as reported for all other neurons investigated previously. Neuronal cultures prepared on laminin- and concanavalin A-coated surfaces also demonstrated dramatic differences in the pattern of axonal growth, dynamics of axonal microtubules, and response to brefeldin A treatment. Our findings suggest that transport and dynamics of axonal microtubules may be directly affected by the mechanical tension produced by growth cone activity.

Key words: tubulin; slow axonal transport; microtubules; photobleaching; neuronal cultures; mechanical tension
A growing axon can be divided into two morphologically and functionally distinct domains, the growth cone and the axonal shaft. The actin-based growth cone is crucial for axonal growth and guidance, whereas the microtubule (MT)-based axonal shaft is specialized for the transport of membrane and cytoskeletal components to the growing axon. The structural subunit of MTs, tubulin, is synthesized in the soma and delivered to the growing axon by active transport. In early experiments using injection of radioactive amino acids in the vicinity of neuronal cell bodies, labeled tubulin was found to be transported in axons at a rate of $1-2 \mathrm{~mm} / \mathrm{d}$ and could be observed in the axoplasm as a coherent peak for a period of weeks (Hoffman and Lasek, 1975; Lasek and Hoffman, 1976; Black and Lasek, 1980). Based on these experiments, it was suggested that tubulin is preassembled into microtubules in the cell body and transported down the axon in the form of MTs.

Strong evidence has been obtained both supporting and opposing the concept of transport of axonal MTs in preassembled form. The concept was most dramatically supported by the observation of anterograde vectorial movement of MTs in Xenopus neurites, as revealed by photoactivation (Reinch et al., 1991; Okabe and Hirokawa, 1992) and photobleaching (Okabe and Hirokawa, 1993) techniques. Experimental evidence on the sites of MT nucleation (Baas and Joshi, 1992; Baas and Ahmad, 1993; Li and Black, 1996; Baas, 1997; Keating et al., 1997) and on the redistribution of MTs from the cell body into distal axonal segments

Received Aug. 28, 1997; revised Oct. 23, 1997; accepted Nov. 6, 1997.

This work was supported by National Institutes of Health Grants NS 33570 to S.V.P. and GM 25062 to G.G.B. We also thank Regeneron Pharmaceuticals, Inc. for generously providing NT-3, BDNF, and CNTF. We are grateful to John Peloquin for preparation of labeled tubulin and to Steve Limbach for maintenance of the digital fluorescence and laser photobleaching system. We thank Primal de Lanerolle, Elly Tanaka, and Mark Rasenick for helpful discussion and comments.

Correspondence should be addressed to Dr. Sergey V. Popov, Department of Physiology and Biophysics (M/C 901), University of Illinois at Chicago, 835 South Wolcott Avenue, Chicago, IL 60612.

Copyright (C) 1998 Society for Neuroscience $0270-6474 / 98 / 180821-09 \$ 05.00 / 0$
(Slaughter et al., 1997) is consistent with the idea that a significant population of axonal MTs is transported down the axon in the assembled form. Conversely, photoactivation and photobleaching methods failed to detect a population of translocating MTs in the axons of sensory neurons (Okabe and Hirokawa, 1990, 1992), pheochromocytoma (PC12) cells (Lim et al., 1990), Ti1 pioneer neurons in living grasshoppers (Sabry et al., 1995), and in living zebrafish (Takeda et al., 1995). These and other data (Bamburg et al., 1986; Funakoshi et al., 1996; Miller and Joshi, 1996) suggest that the majority of axonal tubulin is in the form of nontranslocating but dynamic MTs.

The conflicting results on the pattern of MT transport between Xenopus and the other neurons studied previously represent a paradox needing investigation. In this paper, we show that the pattern of axonal growth as well as turnover rate and translocation of axonal MTs in growing Xenopus neurites depends on the culture conditions. Taken together, our results suggest that critical intrinsic aspects of axonal MT dynamics may be directly controlled by mechanical tension produced by the growth cone and by exogenous factors such as attachment to the substrate.

\section{MATERIALS AND METHODS}

Preparation of Cy3-tubulin. MT protein was prepared from porcine brain by cycles of assembly and disassembly (Borisy et al., 1975). Labeling of porcine brain tubulin with $\mathrm{Cy} 3$ was performed as described previously (Keating et al., 1997). Details of Cy3-tubulin preparation can be obtained from the Borisy Laboratory web site (http://borisy.bocklabs.wisc.edu). Before microinjection, a $10 \mu \mathrm{l}$ aliquot of Cy3-tubulin was centrif uged at $15,000 \times g$ for $30 \mathrm{~min}$ at $4^{\circ} \mathrm{C}$ to remove particulate material and to reduce pipette clogging and was stored on ice until the time of injection.

Microinjection of Cy3-tubulin into Xenopus embryos. Xenopus eggs were fertilized and dejellied in vitro as described previously (Popov and Poo, 1992). At the two cell stage, the eggs were injected with $10-25 \mathrm{nl}$ of 10 $\mathrm{mg} / \mathrm{ml} \mathrm{Cy3-tubulin} \mathrm{using} \mathrm{the} \mathrm{air} \mathrm{pressure} \mathrm{injector} \mathrm{Picospritzer} \mathrm{II} \mathrm{(Gen-}$ eral Valve, Fairfield, NJ). The diameter of the pipettes used for injection ranged from 9 to $18 \mu \mathrm{m}$. Both injection and subsequent incubation of injected embryos were performed in 10\% Ringer's solution (115 mM $\mathrm{NaCl}, 2 \mathrm{~mm} \mathrm{CaCl}_{2}, 2.5 \mathrm{~mm} \mathrm{KCl}$, and $10 \mathrm{~mm}$ HEPES, pH 7.6). The eggs 
were allowed to develop to stages 19-24 and were then used for the preparation of neuronal cultures.

Cell cultures. Xenopus embryo neuronal cultures were prepared according to previously reported methods (Spitzer and Lamborghini, 1976; Popov et al., 1993). Briefly, the neural tube of embryos at stages 19-24 was dissociated in $\mathrm{Ca}^{2+}$ - and $\mathrm{Mg}^{2+}$-free solution (115 mM NaCl, $2.6 \mathrm{~mm}$ $\mathrm{KCl}, 10 \mathrm{~mm}$ HEPES, and $0.4 \mathrm{~mm}$ EDTA, pH 7.6). Dissociated cells were plated on glass coverslips precoated with laminin $\left(2-5 \mu \mathrm{g} / \mathrm{cm}^{2}\right.$; GIBCO) or with Con A $\left(0.1-1.0 \mu \mathrm{g} / \mathrm{cm}^{2}\right.$; Sigma, St. Louis, MO). The cultures were kept at $20^{\circ} \mathrm{C}$ in a culture medium consisting of $50 \%$ (v/v) Ringer's solution, 49\% L-15 Leibovitz medium (GIBCO), and 1\% fetal bovine serum (GIBCO). The neurons were used for experiments 6-36 hr after plating. In some experiments, neurotrophic growth factors neurotrophin 3 (NT-3), ciliary neurotrophic factor (CNTF), and brain-derived neurotrophic factor $(\mathrm{BDNF})(50 \mathrm{ng} / \mathrm{ml}$ each) were added to the culture medium during cell culture preparation. Brefeldin A (Sigma) was prepared as a $5 \mathrm{mg} / \mathrm{ml}$ stock solution in methanol and stored at $-20^{\circ} \mathrm{C}$.

Photobleaching. The apparatus used for photobleaching has been described in detail previously (Gorbsky et al., 1987). Briefly, the beam of a $3 \mathrm{~W}$ argon ion laser (Spectra-Physics, Fremont, CA) was channeled into the epi-illumination system of a Carl Zeiss IM-35 microscope. A $63 \times, 1.4$ NA objective was used, and a cylindrical lens was positioned to produce a focused $4 \times 57 \mu \mathrm{m}$ beam cross-section in the specimen plane. For the photobleaching experiments, the laser was operated at $514 \mathrm{~nm}$ and 200 $\mathrm{mW}$ for $50-200 \mathrm{msec}$. Irradiation at this laser intensity does not disrupt MTs (Gorbsky et al., 1987; Rodionov et al., 1994; Keating et al., 1997).

Image acquisition and data analysis. A Zeiss IM-35-inverted microscope equipped with a $100 \mathrm{~W}$ mercury arc lamp was used for fluorescence microscopy. The light passed through ultraviolet- and infrared-blocking filters, neutral density filters, and a rhodamine wide-band filter. A siliconintensified target video camera (Dage-MTI, Inc., Michigan City, IN) was used for focusing. Images were acquired with a charge-coupled device (CCD) camera (CH250; Photometrics, Tucson, AZ) driven by IPLab (Signal Analitics Corporation, Vienna, VA) imaging software. The CCD camera was thermoelectrically cooled to $-50^{\circ} \mathrm{C}$ to reduce the darkcurrent noise. Exposure time was $1 \mathrm{sec}$, and images were collected at $10-120 \mathrm{sec}$ intervals. Cells were kept at room temperature during photobleaching and observation. Images were processed with IPLab and Photoshop (Adobe Systems, Mountain View, CA). Quantitation of photobleaching data were performed using IPLab software.

\section{RESULTS}

\section{Microtubules translocate anterogradely in neurons plated on a laminin-coated surface}

Cy3-conjugated tubulin was injected into one of the blastomeres of two-cell-stage Xenopus embryos. The embryos were allowed to develop for $1 \mathrm{~d}$ after injection, and then neuronal cultures were prepared on laminin-coated coverslips. The first neurites were detected $\sim 4 \mathrm{hr}$ after plating. Although no data are available on the differentiation of these neurites into axons and dendrites, all processes produced by Xenopus embryonic neurons in culture are usually referred to as "axons" (Reinch et al., 1991; Okabe and Hirokawa, 1992; Popov et al., 1993), a term that we will use below. The average rate of axonal elongation for the period 6-16 hr after plating was $49 \pm 3 \mu \mathrm{m} / \mathrm{hr}$ (mean $\pm \mathrm{SEM} ; n=54)$. Photobleaching experiments were performed $6-36 \mathrm{hr}$ after cell culture preparation on neurons containing fluorescently labeled MTs. A small zone of axon was illuminated with a short (50-200 msec) flash of an argon laser, and the movement of the bleached segment was monitored with a cooled CCD camera. Figure 1 is an example of a typical experiment in which the bleached zone was placed $\sim 40$ $\mu \mathrm{m}$ behind the growth cone. As reported previously for Xenopus neuronal cultures plated on surfaces coated with matrigel (Reinch et al., 1991) or laminin (Okabe and Hirokawa, 1992), the bleached zone rapidly moved forward indicating anterograde vectorial movement of axonal MTs. The rate of bleached zone movement $(48 \mu \mathrm{m} / \mathrm{hr})$ was $\sim 60 \%$ the rate of growth cone advance $(85 \mu \mathrm{m} / \mathrm{hr})$. Fluorescence recovery in the bleached zone was characteristically rapid, limiting the ability to monitor its
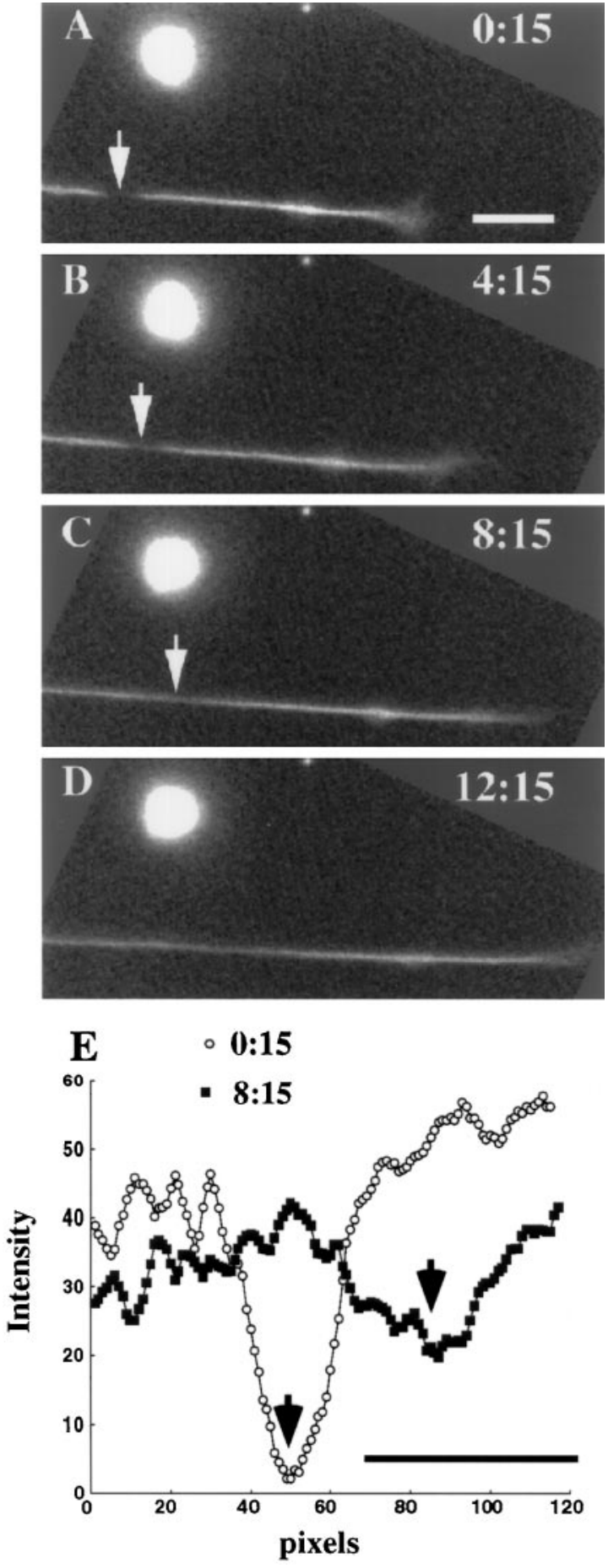

Figure 1. Anterograde movement of photobleached MTs in an elongating Xenopus neurite growing on laminin-coated substrate. $A-D$, Fluorescent images of a neurite captured after photobleaching. The photobleaching was performed $16 \mathrm{hr}$ after cell culture preparation. The bleached segment was at the distal (near the growth cone) axonal segment. Numbers indicate the time in minutes and seconds after the photobleaching pulse. The position of the center of the bleached zone is indicated by arrows. The photobleached zone remained visible for $\sim 6 \mathrm{~min}$. Forward movement of the photobleached zone was clearly visible during the first few minutes after photobleaching. The rate of bleached zone movement was $\sim 48 \mu \mathrm{m} / \mathrm{hr}$, and the rate of growth cone advance was $\sim 85 \mu \mathrm{m} / \mathrm{hr}$. $E$, Fluorescence intensity profiles of an axonal segment including the photobleached segment created from images $A$ (15 sec after photobleaching, open circles) and $C$ ( $8 \mathrm{~min}$ and $15 \mathrm{sec}$ after photobleaching, filled squares). The bottom of the fluorescence intensity profile (arrows) moved toward the distal end of the neurite. Scale bars, $10 \mu \mathrm{m}$. 
movement to 5-10 min. Thus, our results confirm previous reports (Reinch et al., 1991; Okabe and Hirokawa, 1992) that MTs in Xenopus neurons are transported toward the growth cone and are highly dynamic.

\section{Microtubules are stationary in neurons plated on a concanavalin A-coated surface}

The extension of Xenopus neurites on laminin- or matrigel-coated substrata is unusually fast with reported rates up to $200 \mu \mathrm{m} / \mathrm{hr}$ and an average of $\sim 50-80 \mu \mathrm{m} / \mathrm{hr}$ (Reinch et al., 1991; Okabe and Hirokawa, 1992; Popov et al., 1993). The fast axonal growth is likely to impose stringent demands on axonal transport systems. Therefore, it has been repeatedly suggested that the speed and coherence of MT transport in Xenopus axons is likely to be greater than that of other neuronal types (Reinch et al., 1991; Sabry et al., 1995; Takeda et al., 1995; Baas, 1997; Slaughter et al., 1997). This may facilitate detection of MT transport by the photobleaching technique and explain why anterograde MT translocation was observed in Xenopus but not in other neuronal types. However, the growth pattern of Xenopus neurons on laminin-coated surfaces is rather unusual. Neurites are poorly attached to the substrate, seem to be under tension produced by the rapidly advancing growth cone, and decrease their diameter during axonal growth (Okabe and Hirokawa, 1992; Popov et al., 1993). To examine whether the anterograde translocation of axonal MTs that we observed was dependent on adhesion of neurites to the substratum, we prepared cultures of Xenopus embryonic neurons on different surfaces, including clean glass, poly-Llysine, collagen, and concanavalin A (Con A). The rate of axonal growth under these culture conditions varied from a few micrometers per hour (clean glass) to $\sim 30-40 \mu \mathrm{m} / \mathrm{hr}$ (Con A). For the reasons described above, we focused on rapidly growing neurites on Con A-coated coverslips. Under these culture conditions, the first neurites were detected $\sim 4-6 \mathrm{hr}$ after cell culture preparation. The average rate of axonal elongation for the period $6-16 \mathrm{hr}$ after plating was $31 \pm 3 \mu \mathrm{m} / \mathrm{hr}$ (mean $\pm \mathrm{SEM} ; n=39$ ). Photobleaching experiments were performed 8-36 hr after cell culture preparation on rapidly extending and relatively long ( $>500 \mu \mathrm{m}$ in length) neurites. The optical system used for photobleaching and visualization of the bleached zone movement, as well as intensity of laser irradiation and duration of the laser flash, was identical to those used in experiments performed on neurons growing on laminin-coated substrate. Figure 2 shows a typical example of a photobleaching experiment. During a $30 \mathrm{~min}$ period after photobleaching, the neurite elongated by $\sim 20 \mu \mathrm{m}$. The bleached zone was clearly visible for the duration of experiment. The position of the bleached zone remained constant relative to the substrate throughout the experimental run, a conclusion supported by analysis of the fluorescence profiles (Fig. 2E). We estimate that the accuracy of the measurement of position of the bleached zone was $\sim 1 \mu \mathrm{m}$, and therefore the rate of the bleached zone movement was $<2 \mu \mathrm{m} / \mathrm{hr}$.

Although some of the neurites on Con A-coated substrate grew rapidly $(>60 \mu \mathrm{m} / \mathrm{hr}$ ), the average rate of axonal elongation on a Con A-coated surface was significantly lower than that on laminin. To investigate whether the qualitatively different pattern of MT translocation on laminin- and Con A-coated surfaces is directly related to the difference in the rates of axonal growth, we repeated the photobleaching experiments on neurons plated on Con A-coated substrate in the presence of a cocktail of neurotrophic growth factors, NT-3, CNTF, and BDNF (50 ng/ml each), in the culture medium. These neurotrophic factors are
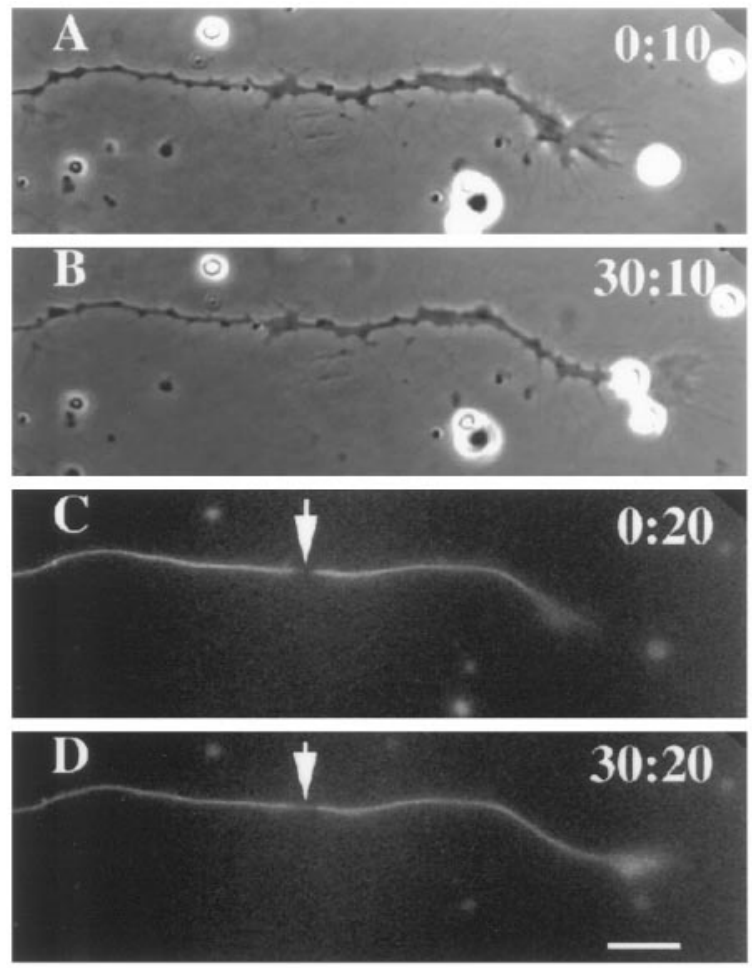

E

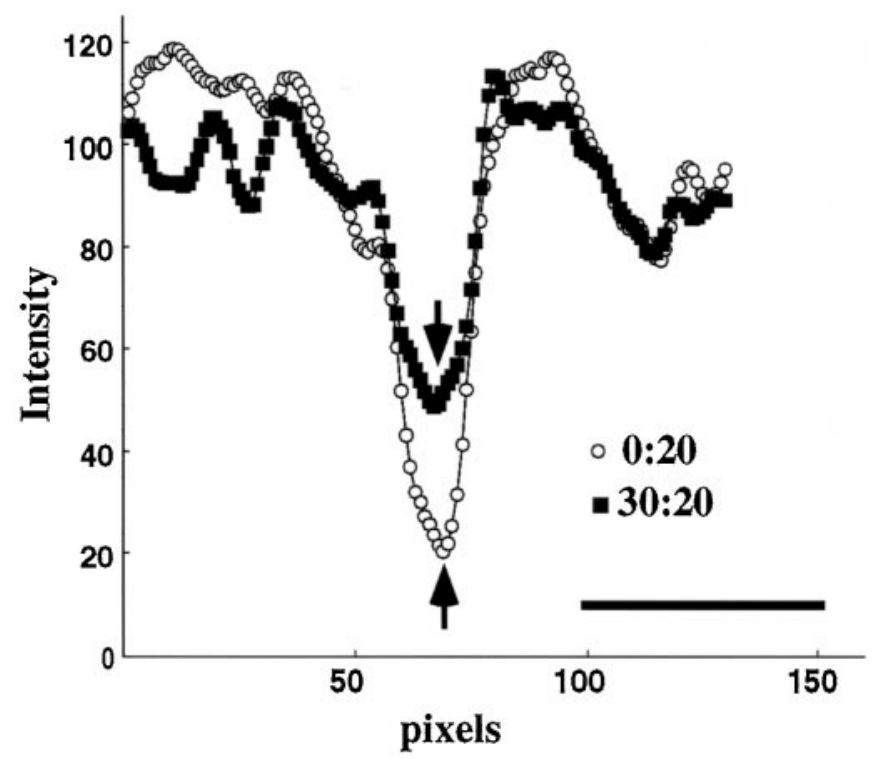

Figure 2. MTs remain stationary during axonal growth on Con A-coated substrate. $A-D$, Phase contrast $(A, B)$ and fluorescent $(C, D)$ images of a neurite $12 \mathrm{hr}$ after cell culture preparation. The time after photobleaching is indicated in minutes and seconds in the top right corner of each panel. The bleached zone was clearly visible during the experiment (30 min). No obvious change in the position of the bleached segment was observed. The neurite continued to grow and extended by $\sim 20 \mu \mathrm{m}$. E, Fluorescence intensity profiles of an axonal segment including the photobleached segment created from images $C$ (20 sec after photobleaching, open circles) and $D$ (30 min and $20 \mathrm{sec}$ after photobleaching, filled squares). The bottom of the fluorescence intensity profile (arrows) remained stationary within experimental error $(\sim 1 \mu \mathrm{m})$. Scale bars, $10 \mu \mathrm{m}$. 

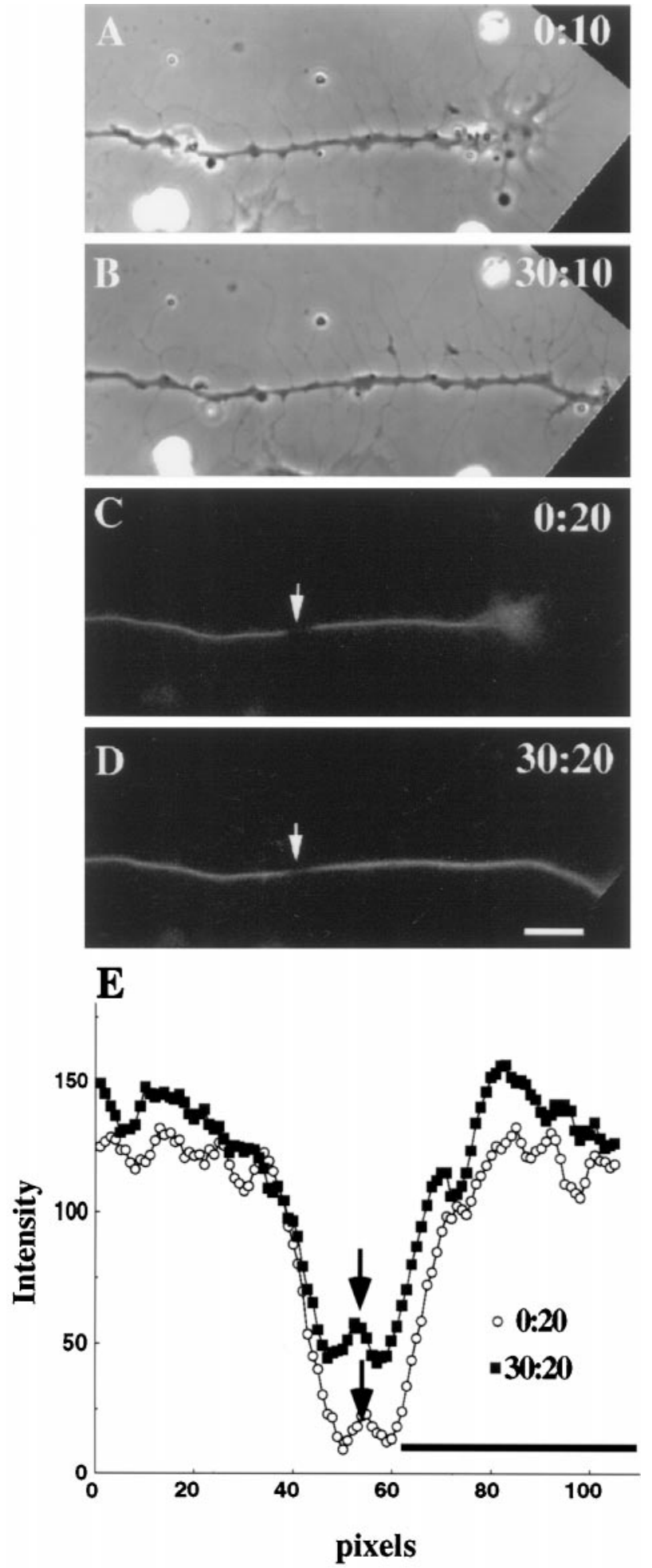

Figure 3. MTs remain stationary in rapidly growing neurites plated on a Con A-coated surface in the presence of neurotrophic factors. $A-D$, Phase contrast $(A, B)$ and fluorescent $(C, D)$ images of a neurite growing in the presence of neurotrophic factors NT-3, BDNF, and CNTF $(50 \mathrm{ng} / \mathrm{ml}$ each) in the culture medium. The photobleaching was performed $18 \mathrm{hr}$ after cell culture preparation. The time after photobleaching is indicated in minutes and seconds in the top right corner of each panel. The length of the neurite was $\sim 900 \mu \mathrm{m}$, and the rate of axonal growth was $\sim 57 \mu \mathrm{m} / \mathrm{hr}$. The photobleached zone was clearly visible during the experiment $(30$ $\mathrm{min}$ ) and remained stationary within experimental error $(\sim 1 \mu \mathrm{m})$ (arrows in $C$ and $D$ ). E, Fluorescence intensity profiles of an axonal known to promote survival and differentiation of Xenopus embryonic neurons in culture (Lohof et al., 1993; Stoop and Poo, 1995; Wang et al., 1995). In the presence of neurotrophic factors, some of the neurites grew very rapidly $(>100 \mu \mathrm{m} / \mathrm{hr})$ and $16 \mathrm{hr}$ after plating exceeded $1100 \mu \mathrm{m}$ in length. The average rate of axonal growth on Con A-coated substrate was significantly higher in the presence $(57 \pm 3 \mu \mathrm{m} / \mathrm{hr}$, mean $\pm \mathrm{SEM} ; n=57)$ than in the absence $(31 \pm 3 \mu \mathrm{m} / \mathrm{hr}$, mean $\pm \mathrm{SEM} ; n=39)$ of neurotrophins $(p<0.001, t$ test) and was similar to the rate of axonal growth on laminin-coated substrate $(49 \pm 3 \mu \mathrm{m} / \mathrm{hr})$. Figure 3 shows an example of a photobleaching experiment performed on a neurite growing rapidly in the presence of neurotrophins on a Con A-coated substrate. The length of the neurite was $\sim 900 \mu \mathrm{m}$. The photobleached zone was placed on the distal part of an axon. No significant movement of the photobleached zone was observed throughout the experimental run (Fig. $3 C-E$ ), although the axon elongated rapidly and the increase in axonal length was $\sim 28 \mu \mathrm{m}$ during the $30 \mathrm{~min}$ observation period.

\section{Quantitative assessment of movement and fluorescence recovery of bleached zones}

In total, we analyzed 18 photobleached zones in 15 neurites on a laminin-coated surface and 25 photobleached zones in 21 neurites on a Con A-coated surface. The majority of the neurites (31 of 36) were growing, and five were essentially stationary during the experimental run (typically 20-40 min). All neurites (including the stationary ones) were tipped with active growth cones. Neurites chosen for photobleaching experiments grew with an average rate of $59 \pm 7 \mu \mathrm{m} / \mathrm{hr}($ mean \pm SEM) and $32 \pm 4 \mu \mathrm{m} / \mathrm{hr}$ (mean \pm SEM) for laminin- and Con A-coated substrata, respectively. In each experiment, we measured the rate of axonal elongation and the rate and direction of translocation of the center of the bleached segment, which reflects net movement of tubulin polymer. In Figure 4, movement of bleached zones relative to the substrate is plotted against axonal growth rate. In all neurites growing on laminin-coated substrate, anterograde movement of the bleached zone was observed (Fig. $4 A$ ). In general, the rate of MT movement was somewhat smaller than that of axonal growth. The average rate of bleached zone movement was $32 \pm$ $5 \mu \mathrm{m} / \mathrm{hr}$ (mean $\pm \mathrm{SEM} ; n=18$ ). In one case (marked by an arrow), we observed rapid anterograde movement of MTs in a stationary neurite. In neurites growing on Con A-coated substrate (Fig. $4 B$ ), the bleached zones were stationary within experimental error regardless of the rate of axonal growth. The average rate of bleached zone movement was $0.0 \pm 0.5 \mu \mathrm{m} / \mathrm{hr}$ (mean \pm SEM; $n=25$ ), suggesting that the majority of axonal MTs in neurites growing on Con A-coated substrate are primarily stationary.

To investigate the dynamics of axonal MTs in growing neurites, we measured the half-time of fluorescence recovery of the bleached segment $\left(t_{1 / 2}\right)$, usually considered an indicator of MT turnover rate (Okabe and Hirokawa, 1990; Edson et al., 1993). Both for laminin- and Con A-coated substrata, the data were divided into two categories: (1) activation of distal axonal segments (within $100 \mu \mathrm{m}$ of the growth cone) and (2) activation of

$\leftarrow$

segment including the pho-tobleached segment created from images $C$ (20 sec after photobleaching, open circles) and $D$ (30 min and $20 \mathrm{sec}$ after photobleaching, filled squares). The bottom of the fluorescence intensity profile (arrows) remained stationary within experimental error $(\sim 1 \mu \mathrm{m})$. Scale bars, $10 \mu \mathrm{m}$. 

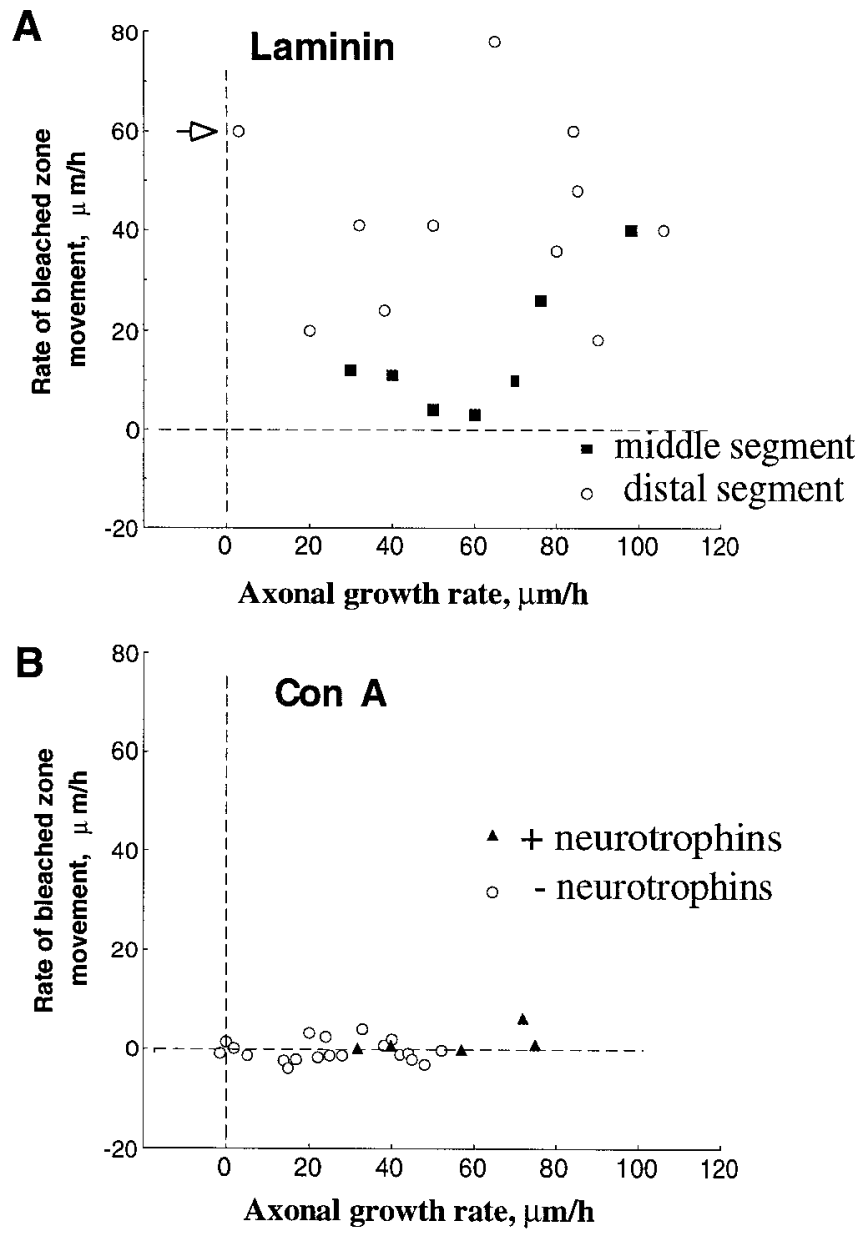

Figure 4. Quantitative assessment of the movement of bleached zones. The rate of the movement of the center of the bleached zone relative to the substrate is plotted as a function of neurite elongation rate. $A$, Neurites growing on laminin-coated substrate. The average rate of neurite extension was $59 \pm 7 \mu \mathrm{m} / \mathrm{hr}$ (mean $\pm \mathrm{SEM} ; n=18$ ). The bleached zone was located at the distal axonal segment (open circles) and at the proximal segment ( filled squares) in 11 and 7 experiments, respectively. The average rate of bleached zone movement was $32 \pm 5 \mu \mathrm{m} / \mathrm{hr}$ (mean $\pm \mathrm{SEM}$ ). In each experiment, the accuracy of the measurements of the positions of growth cone and bleached zone was $\sim 1 \mu \mathrm{m}$. Detection of the MT translocation rate was more accurate for neurites with relatively stable MTs (slow fluorescence recovery). Generally the bleached zone could be reliably traced for a period of 10-20 min, and therefore the accuracy of the measurements was $\sim 3-6 \mu \mathrm{m} / \mathrm{hr}$. In seven experiments, we were not able to measure reliably the rate of bleached zone movement. In two of these seven cases, the bleached zone was visible only for a few minutes $\left(t \frac{1}{2}\right.$ of $\left.\sim 3 \mathrm{~min}\right)$, precluding accurate measurements of the MT movement. In the remaining five cases, the lateral movement of the whole axonal structure was very fast, and the measurements of the bleached zone position were meaningless. Results of these seven experiments have been excluded from the analysis of MT movement. $B$, Neurites growing on Con A-coated substrate; summary of 25 different experiments. In five experiments (filled triangles), neurotrophic factors NT-3, BDNF, and CNTF $(50 \mathrm{ng} / \mathrm{ml}$ each) were added to the culture medium during cell culture preparation and were present throughout the experiment. No neurotrophic factors were added to the culture medium in the remaining 20 experiments (open circles). The bleached zone was at the distal axonal segment in 18 experiments and at the proximal segment in seven experiments. In the absence of NT-3 in the culture medium, the average rate of axonal growth and the rate of bleached zone movement were $25.6 \pm 3.7$ and $-0.4 \pm 0.5 \mu \mathrm{m} / \mathrm{hr}$, respectively (mean \pm SEM; $n=20$ ). In the presence of NT-3, the rates of axonal growth and the bleached zone movement were $55 \pm 8$ and $1.4 \pm 1.2 \mu \mathrm{m} / \mathrm{hr}$, respectively (mean $\pm \mathrm{SEM} ; n=5$ ). The position of the bleached zone could be measured with an accuracy of $\sim 1 \mu \mathrm{m}$. Typically the movement of the bleached zone was followed for 30-60 min. Therefore we estimate the accuracy of the measurements of the MT movement rate to be $\sim 1-2 \mu \mathrm{m} / \mathrm{hr}$.

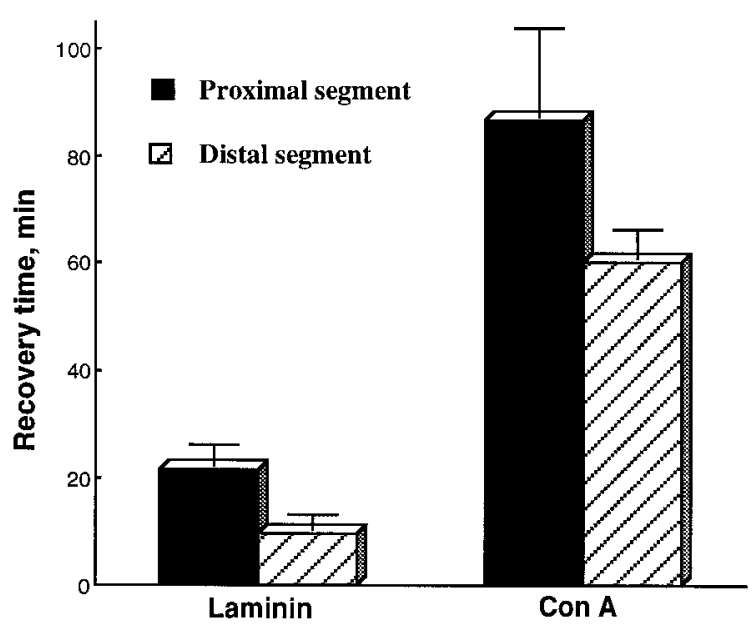

Figure 5. Quantitative analysis of the fluorescence recovery in the bleached zones. Experiments were performed as described in Figures 1-3. The bleached zones were at the proximal (black bars) or distal (hatched bars) axonal segments. In each experiment, the average time for $50 \%$ recovery of fluorescence $(t / 2)$ was calculated. For neuronal cultures growing on either laminin- or Con A-coated substrate, $t \frac{1}{2}$ values were significantly higher at the proximal segments compared with the distal segments. Recovery of fluorescence was approximately fivefold faster (smaller $t_{1 / 2}$ values) in neurites growing on laminin-coated compared with those growing on Con A-coated substrate.

proximal segments ( $>300 \mu \mathrm{m}$ from the growth cone). For the neurites growing on laminin, $t_{1 / 2}$ values were $21.5 \pm 4.0 \mathrm{~min}$ (mean $\pm \mathrm{SEM} ; n=7)$ and $9.7 \pm 2.7 \mathrm{~min}(n=11)$ for the proximal and distal axonal segments, respectively. On Con A-coated substrate, $t_{1 / 2}$ values were $86.7 \pm 16.3 \min (n=7)$ and $60.0 \pm 5.4 \mathrm{~min}(n=18)$ for the proximal and distal segments, respectively (Fig. 5). Both for distal and proximal segments, recovery of fluorescence after photobleaching was approximately fivefold faster in neurites plated on laminin-coated substrate than in those growing on Con A $(p<0.001, t$ test), suggesting significantly slower turnover of axonal MTs on Con A-treated substrate. In agreement with previously published data (Edson et al., 1993), recovery of photobleaching in the distal axonal segments was significantly faster than that in the proximal segments $(p<0.05, t$ test) for neuronal cultures plated on either laminin or Con A, indicating slower MT turnover rates in the proximal axonal segments. However, it should be noted that the apparent faster rate of MT turnover in neurites growing on laminin may be related to asynchronous translocation of MTs relative to each other (see Discussion).

In summary, axonal MTs in neurites growing on laminin- or Con A-coated substrate differ both qualitatively and quantitatively in the pattern of their movement. In neurites growing on laminin-coated substrate, MTs rapidly translocate forward with a rate comparable with that of axonal elongation and seem to be highly dynamic. In neurites growing on Con A-coated substrate, MTs are significantly more stable and stationary regardless of the axonal growth rate. The observed differences in the MT movement were not related to the different axonal lengths or rates of axonal growth on the two substrata.

\section{Differential pattern of axonal growth on laminin- and Con A-treated substrata}

To determine whether differential behavior of axonal MTs on laminin- and Con A-coated surfaces may be related to the different patterns of axonal growth, we characterized the morphology of 
neuronal cells on both substrata. Both on laminin- or Con A-coated surfaces, neurons projected long neurites tipped with highly active growth cones. On both surfaces, the neurites grew rapidly. In agreement with previously published data (Reinch et al., 1991; Okabe and Hirokawa, 1992; Popov et al., 1993), we found that on a laminin-coated surface the neurites are often straight and attached to the substrate only at the growth cone region. The attachments along the axonal shaft, if any, are transient and readily disrupted during neurite extension. In the cases in which the neurites were attached to the substrate along their length, the shape of the axon was changing constantly, and the position of the branching points was not fixed either in relation to the substrate or in relation to the neurite. At the later stages of growth, the neurites were visibly thinner than they were at the initial stages of growth, indicating stretching of the whole axonal structure (Okabe and Hirokawa, 1992; Popov et al., 1993). In vitro growth rates of these neurons are a few fold higher than are the growth rates of the same neurons in vivo (Jacobson and Huang, 1985).

On the contrary, when plated on a Con A-coated surface, the axons were firmly attached to the substrate. During neurite elongation, the proximal segments did not change their position relative to the substrate, and axonal diameter did not change noticeably. The position of the branch points and the distance between the branch points also did not change. Furthermore, the distance between individual filopodia-like processes formed along the axonal shaft also remained constant (Fig. 6, see also Figs. 2, 3). Neither the pattern of axonal growth on Con A-coated substrate nor axonal morphology were affected by the presence of neurotrophic factors in the culture medium (data not shown). The relatively stable position of the proximal axonal segments and branch points in relation to the substrate during axonal growth is routinely observed both in vitro (Lim et al., 1990; Craig et al., 1995) and in vivo (Sabry et al., 1995; Takeda et al., 1995) and is likely to be a general feature of axonal growth mediated by growth cone activity.

\section{Differential dependence on the supply of new membrane}

To study how axonal elongation in neuronal cultures plated on laminin- and Con A-coated surfaces depends on the supply of new membrane material synthesized in the cell body, we treated the cultures with Brefeldin A, a drug that blocks membrane traffic through the Golgi complex (Lippincott-Schwartz et al., 1989 , 1990). A typical response of neurites growing on a Con A-coated substrate is illustrated in Figure $7 A$. After Brefeldin A (BFA) application, elongation of the neurite slowed within a few minutes, and the growth cone retracted within $12 \mathrm{~min}$ (Fig. 7A). In total, we followed the growth of 16 neurites on Con A-coated substrate after Brefeldin A treatment. Within $1 \mathrm{hr}$ after Brefeldin A application, the advance of 14 of the 16 neurites almost completely stopped. In the two instances in which axonal growth persisted for $>1 \mathrm{hr}$ after Brefeldin A treatment, the neurites became visibly thinner (data not shown). In contrast, growth continued on laminin although at a somewhat reduced rate (Fig. $7 B, C)$. None of the 20 neurites investigated stopped elongating within $1 \mathrm{hr}$ of the treatment. Axonal elongation on laminincoated surfaces continued for a period of up to $8 \mathrm{hr}$ with an average duration of $275 \pm 30 \mathrm{~min}$ (mean \pm SEM). To characterize quantitatively the effects of BFA on axonal growth, we plotted the rate of axonal growth as a function of time after the onset of BFA application (Fig. 8). The rate of axonal elongation on Con A decreased twofold $\sim 7$ min after BFA application, and the growth
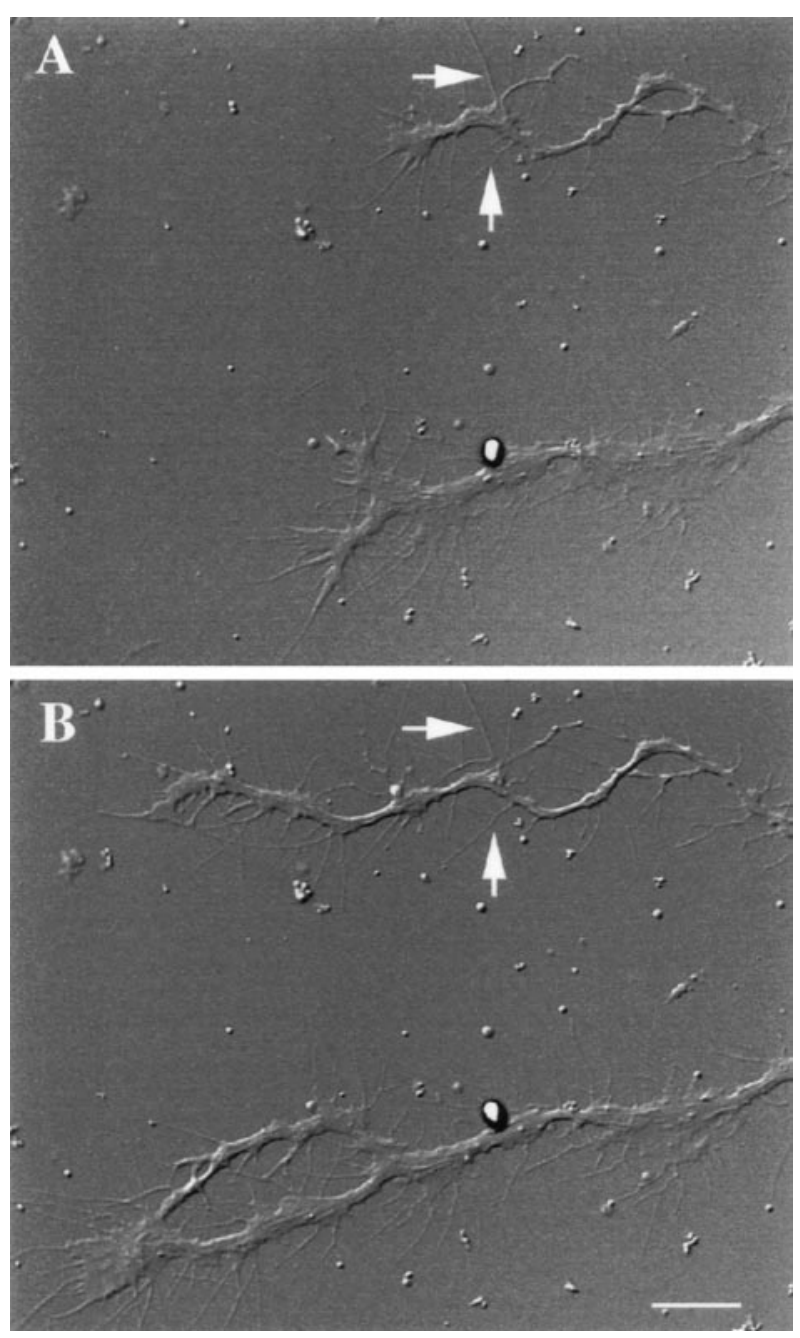

Figure 6. Axonal growth on Con A-coated substrate. $A, B$, DIC images of neurites growing on Con A-coated substrate. The image $B$ was taken 2 hr and 15 min after image $A$. Notice the constant position of the axon, branch point, and filopodia-like processes (arrows) relative to the substrate. Scale bar, $20 \mu \mathrm{m}$.

almost completely terminated in $\sim 60$ min (Fig. $8 A$ ). The rate of axonal growth on laminin-coated substrate slowed after BFA application and decreased twofold $\sim 90$ min after the start of BFA treatment (Fig. 8B). However, axonal elongation continued for a period of up to $8 \mathrm{hr}$.

\section{DISCUSSION}

Formation and maintenance of axonal MTs depend on the transport of tubulin from the cell body to the axon. The structural hypothesis of slow axonal transport holds that tubulin moves down the axon in the form of MTs (Hoffman and Lasek, 1975; Lasek and Hoffman, 1976; Baas, 1997). A seemingly direct way to test this hypothesis is to create a reference mark on the MTs by photobleaching or photoactivation and to determine whether the mark moves. Experiments in various types of neuronal cells demonstrated that most axonal MTs are primarily stationary. However, in striking contrast to these observations, rapid translocation of axonal MTs down the neurite at the rate of slow axonal transport was observed in Xenopus embryonic neurons (Reinch et al., 1991). It has been repeatedly argued that because of the high rate of axonal growth, MT transport in Xenopus 

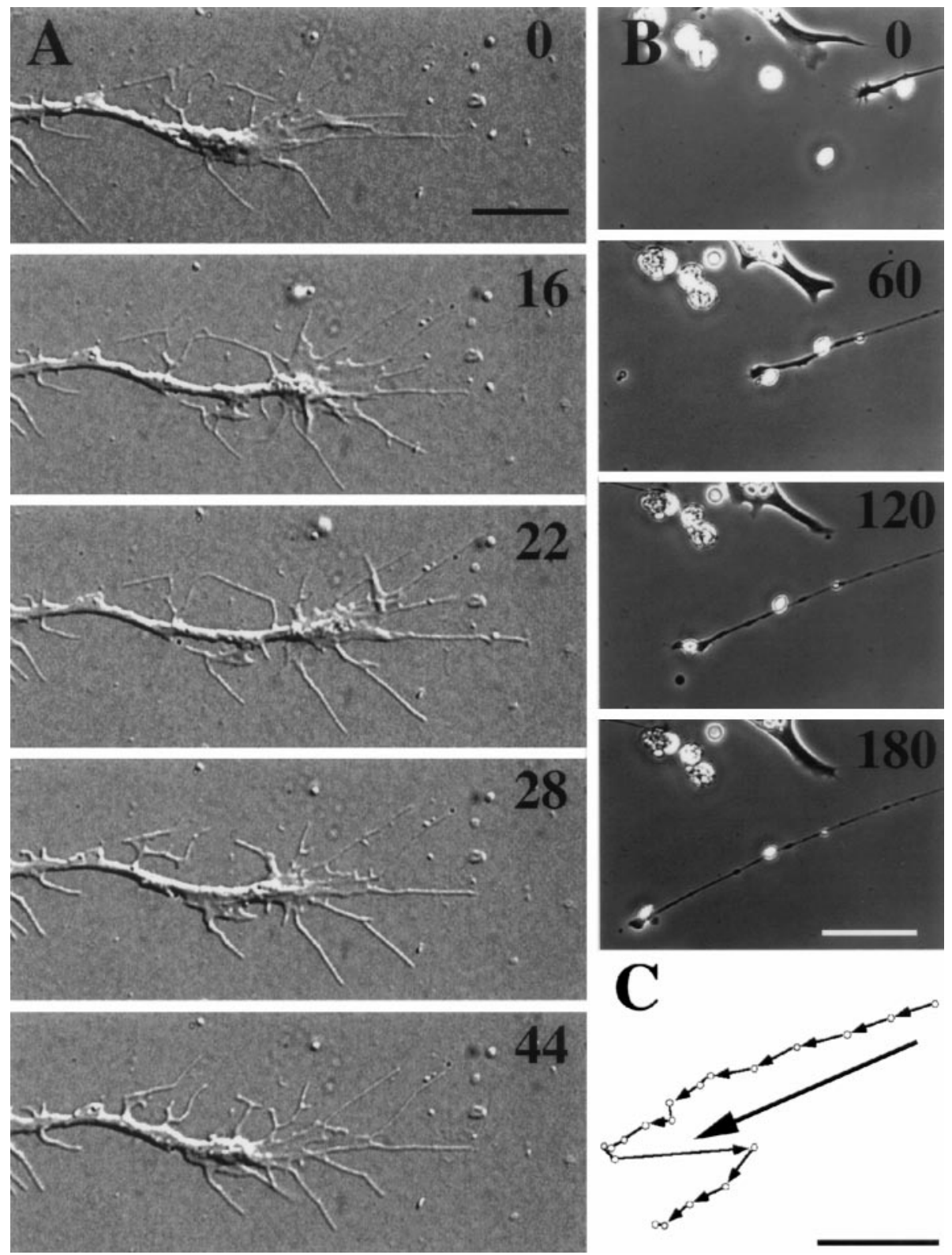

Figure 7. Effect of Brefeldin A treatment on axonal growth. $A$, DIC images of a neurite growing on Con A-coated substrate. Numbers indicate time in minutes. Brefeldin A $(10 \mu \mathrm{g} /$ $\mathrm{ml})$ was applied $16 \mathrm{~min}$ after the start of an experiment. Elongation of the neurite after BFA application visibly slowed down, and the growth cone retracted within $12 \mathrm{~min}$ after drug treatment $(28 \mathrm{~min}$ after the start of experiment). $B, C$, Neurite growth on laminin-coated substrate after Brefeldin A treatment. $B$, Phase contrast images of a neurite. Numbers indicate time in minutes after Brefeldin A $(10 \mu \mathrm{g} / \mathrm{ml})$ application. Axonal growth continues for at least $3 \mathrm{hr}$ after drug treatment. $C$, Quantitative analysis of axonal elongation; data from $B$. The growth cone position relative to the substrate is plotted in 20 min intervals (open circles). The average direction of axonal growth is indicated by a big arrow. The growth cone elongation appears to slow down within 80 min after application of BFA. Approximately $5 \mathrm{hr}$ after drug application, the growth cone retracts. However, elongation resumed and continued for $\sim 80 \mathrm{~min}$, after which the neurite retracted. Scale bars: $A, 10 \mu \mathrm{m} ; B, C, 40 \mu \mathrm{m}$. neurites may be more robust and easily detectable compared with that in other neuronal types. Do Xenopus embryonic neurons really possess a uniquely efficient system for MT transport? Resolution of this problem is important for our understanding of the mechanisms of slow axonal transport.

\section{Axonal growth on laminin- and Con A-coated substrata}

Our results as well as previous studies (Okabe and Hirokawa, 1992; Popov et al., 1993) clearly demonstrate that Xenopus neurites elongating on laminin are attached to the substrate primarily at the soma and the growth cone region and become visibly thinner during elongation. Axonal elongation cannot rely on stretching alone for a considerable period of time and must be supported by a supply of new membrane from the soma. Previous quantitative analysis of the anterograde flow of plasma membrane lipids in Xenopus neurites indicated that new membrane necessary for growth is inserted along the axons as well as at the soma (Popov et al., 1993). Therefore, elongation of Xenopus axons on laminin can be considered a combination of true axonal growth, which depends on the supply of new membrane, and stretching of the axon. This model predicts that axonal elongation would continue for some time (although probably at a slower rate) even in conditions when delivery of Golgi-derived vesicles to the axon is inhibited. Results of our experiments with BFA are totally consistent with this prediction (Figs. $7 B, C, 8 B$ ). On the contrary, neurites growing on Con A-treated coverslips are firmly attached to the substrate and seem to grow by a more conventional mechanism, common to other neuronal types. Their growth depends on the supply of new membrane from the soma, similar to other neurons (Martenson et al., 1993; Dai and Sheetz, 1995; Futerman and Banker, 1996).

\section{Microtubule transport on laminin- and Con A-coated substrata}

Our results obtained in Xenopus neurons plated on laminincoated surfaces are in full agreement with previous reports on the anterograde movement of axonal MTs in these neurons using 


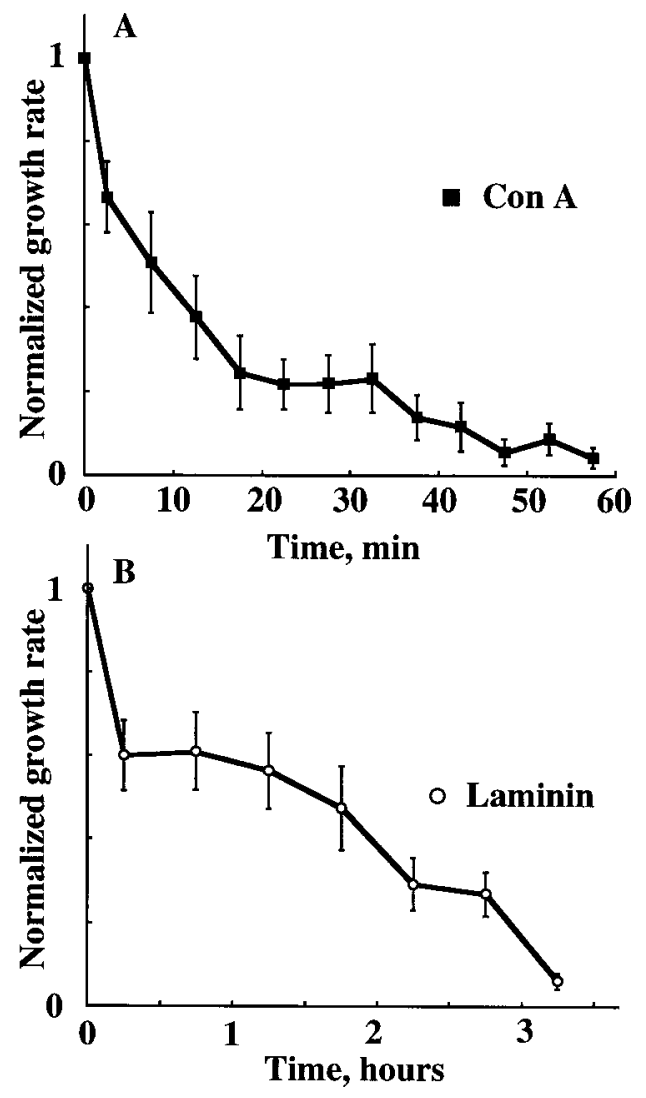

Figure 8. Quantitative analysis of the effects of BFA on axonal growth on Con A-coated $(A)$ and laminin-coated $(B)$ surfaces. In each experiment, the average rate of axonal growth before BFA application $(10 \mu \mathrm{g} / \mathrm{ml})$ was determined for a period of 20-30 min. The rate of axonal elongation was measured as a function of time after the onset of BFA application and was normalized to that before BFA application. Each point represents the mean \pm SEM of $14(A)$ and $20(B)$ different experiments. For neurites growing on Con A-coated substrate in 2 of 16 experiments, we observed a decrease in axonal diameter a few minutes after BFA application. These neurites continued to grow for at least $2 \mathrm{hr}$ and became progressively thinner. They were excluded from the analysis.

photobleaching or photoactivation techniques (Reinch et al., 1991; Okabe and Hirokawa, 1992, 1993). Briefly, transport of axonal MTs in Xenopus neurites differed from other neuronal cell types in a few important respects. (1) MTs seemed to translocate anterogradely en bloc, (2) no stationary population of MTs was observed, and (3) the rate of MT transport correlated with the rate of axonal growth and increased with increasing distance from the soma. One factor that may directly contribute to the anterograde movement of MTs is poor adhesion of neurites growing on laminin to the substrate. In agreement with this hypothesis, when neuronal cultures were prepared on Con A-coated substrate, the MTs were primarily stationary, similar to reports on the stationary nature of axonal MTs in other neuronal types. The only difference between the series of experiments performed on $\mathrm{Xe}$ nopus neurites plated on laminin- and Con A-coated substrata was the nature of the substrate. All other parameters such as preparation of fluorescently tagged tubulin, loading of tubulin into neurons, and parameters of the optical system used for photobleaching and observation of MT dynamics were identical. Therefore it is likely that the differences observed in the movement of the bleached segment in neuronal cultures prepared on two different substrata reflect a differential mode of MT move- ment under these culture conditions, rather than an experimental artifact such as photodamage of MTs. The simplest explanation of our data is that anterograde translocation of axonal microtubules in Xenopus neurites growing on laminin is a direct consequence of axonal stretching and is not related to the activity of the slow axonal transport system. The stretching of the axon, combined with a progressive decrease in axonal diameter, is sufficient to explain the most striking features of MT behavior on the laminin-coated substrate, specifically, the translocation of MTs relative to the substrate and the absence of a stationary population of MTs. The degree of axonal stretching is dictated by the mechanical tension produced by the growth cone (see below) and by the attachment of the axonal shaft to the substrate. The relatively poor correlation between the rates of axonal elongation and MT movement (Fig. 4) is likely to be related to the frequent changes in the pattern of axonal adhesion to the substrate.

The lack of bleached zone movement in neurites growing on a Con A-coated substrate indicates that axonal MTs in Xenopus neurites are primarily stationary regardless of axonal growth rate (Fig. 4). However, a small fraction of transported MTs can escape detection, especially if movement of these MTs is asynchronous. Quantitative analysis of the fluorescence profiles after photoactivation demonstrated that it is impossible to detect a moving population of MTs that represents $10 \%$ or less of the MTs at a given axonal region (Sabry et al., 1995). Therefore, negative results obtained with the photobleaching method in our experiments on Xenopus neurons growing on Con A, as well as in a variety of other neuronal cells, do not exclude the possibility that a small fraction of tubulin is transported in the form of MTs (Ahmad and Baas, 1995; Moritz et al., 1995; Zheng et al., 1995; Keating et al., 1997). However, these results impose an upper limit on the size of this fraction.

\section{Control of microtubule movement and dynamics by mechanical tension}

Mechanical tension is an important intrinsic regulator of axonal growth and development. A case in point is de novo initiation and elongation of pre-existing neurites by experimentally applied mechanical tension (Bray, 1984; Zheng et al., 1991), "towed" axonal growth. In this experimental paradigm, the rate of axonal elongation is proportional to the magnitude of mechanical tension (Lamoureux et al., 1989), suggesting regulation of MT transport and assembly by mechanical tension. Transport of tubulin has not been studied in detail in these neurites. However, because large membrane-attached particles translocate forward during the experimental towed-growth regime (Zheng et al., 1991), it is likely that the whole neurite, including axonal MTs, is towed forward by the experimentally applied mechanical force (Okabe and Hirokawa, 1992). Interestingly, the MT array observed in tension-induced neurites is similar to that in neurites extending by growth cone activity and requires new MT assembly (Zheng et al., 1993). Therefore, it seems that MT transport in this system is a combination of conventional slow axonal transport and anterograde movement induced by stretching. Mechanically, the pattern of Xenopus axonal growth on a laminin-coated substrate is identical to that of neurites growing in response to experimentally applied mechanical tension (Bray, 1984; Zheng et al., 1991) and is likely to occur by a similar mechanism (Tanaka et al., 1995).

We observed about a fivefold difference in the rate of fluorescence recovery of the bleached zone between neurites growing on laminin and Con A. It is generally believed that the rate of fluorescence recovery reflects MT turnover rate. How can this 
intrinsic property of MTs be affected by an extrinsic factor, such as culture substrate? During elongation of Xenopus neurites on laminin, mechanical force applied to the growth cone is not balanced by the adhesion of the axonal shaft to the substrate and transduced to the proximal axonal segments. The mechanical tension along the axonal shaft may directly affect dynamics of axonal MTs. The long-range transduction of cytomechanical forces from the growth cone to proximal axonal regions may also affect other aspects of axonal mechanics and transport. However, it should be noted that asynchronous movement of MTs relative to each other may contribute to fluorescence recovery of the bleached zone as well. Although this MT transport is not related to the turnover of MTs per se, it will lead to a faster rate of fluorescence recovery of the bleached zone.

In conditions in which neurites are firmly attached to the substrate, mechanical tension produced by the growth cone is balanced by the sufficiently strong attachment of neurites to the substrate. It remains to be established whether the long-range signaling between plasma membrane and cytoskeletal elements observed in non-neuronal cells (Ingber, 1997; Maniotis et al., 1997) occurs in axons. However, it is tempting to speculate that mechanical tension may serve as a general regulator of other aspects of axonal growth, such as axonal transport, addition of new membrane to the growing neurite, and local assembly and disassembly dynamics of axonal MTs.

\section{REFERENCES}

Ahmad FJ, Baas PW (1995) Microtubules released from the neuronal centrosomes are transported into the axon. J Cell Sci 108:2761-2769.

Baas PW (1997) Microtubules and axonal growth. Curr Opin Cell Biol 9:29-36.

Baas PW, Ahmad FJ (1993) The transport properties of axonal microtubules establish their polarity orientation. J Cell Biol 120:1427-1437.

Baas PW, Joshi HC (1992) $\gamma$-Tubulin distribution in the neuron: implications for the origins of neurite microtubules. J Cell Biol 119:171-178.

Bamburg JR, Bray D, Chapman K (1986) Assembly of microtubules at the tip of growing axons. Nature 321:788-790.

Black MM, Lasek RJ (1980) Slow components of axonal transport: two cytoskeletal networks. J Cell Biol 86:616-623.

Borisy GG, Marcum JM, Olmsted JB, Murphy DB, Johnson KA (1975) Purification of tubulin and associated high molecular weight proteins from porcine brain and characterization of microtubule assembly in vitro. Ann NY Acad Sci 253:107-132.

Bray D (1984) Axonal growth in response to experimentally applied mechanical tension. Dev Biol 102:379-389.

Craig AM, Wyborski RJ, Banker G (1995) Preferential addition of newly synthesized membrane protein at axonal growth cones. Nature 375:592-594.

Dai DJ, Sheetz MP (1995) Axon membrane flows from the growth cone to the cell body. Cell 83:693-701.

Edson KJ, Lim S-S, Borisy GG, Letourneau PC (1993) FRAP analysis of the stability of the microtubule population along the neurites of chick sensory neurons. Cell Motil Cytoskeleton 25:59-72.

Funakoshi T, Takeda S, Hirokawa N (1996) Active transport of photoactivated tubulin molecules in growing axons revealed by new electron microscopic analysis. J Cell Biol 133:1347-1354.

Futerman AH, Banker GA (1996) The economics of neurite outgrowththe addition of new membrane to growing axons. Trends Neurosci 19:144-149.

Gorbsky GJ, Sammak PJ, Borisy GG (1987) Chromosomes move poleward in anaphase along stationary microtubules that coordinately disassemble from their kinetochore ends. J Cell Biol 104:9-18.

Hoffman PN, Lasek RJ (1975) The slow component of axonal transport: identification of major structural polypeptides of the axon and their generality among mammalian neurons. J Cell Biol 66:351-366.

Ingber DE (1997) Tensegrity: the architectural basis of cellular mechanotransduction. Annu Rev Physiol 59:575-599.

Jacobson M, Huang S (1985) Neurite outgrowth traced by means of horseradish peroxidase inherited from neuronal ancestral cells in frog embryos. Dev Biol 110:102-113.
Keating TJ, Peloquin JG, Rodionov VI, Momcilovic D, Borisy GG (1997) Microtubule release from the centrosome. Proc Natl Acad Sci USA 94:5078-5083.

Lamoureux P, Buxbaum RE, Heidemann SR (1989) Direct evidence that growth cones pull. Nature 340:159-162.

Lasek RJ, Hoffman PN (1976) The neuronal cytoskeleton, axonal transport and axonal growth. Cell Motil 3:1021-1049.

Li Y, Black MM (1996) Microtubule assembly and turnover in growing axons. J Neurosci 16:531-544.

Lim S-S, Edson KJ, Letourneau PC, Borisy GG (1990) A test of microtubule translocation during neurite elongation. J Cell Biol 111:123-130.

Lippincott-Schwartz J, Yuan L, Bonifacino J, Klausner R (1989) Rapid redistribution of Golgi proteins into the ER in cells treated with brefeldin A: evidence for membrane cycling from Golgi to ER. Cell $56: 801-813$

Lippincott-Schwartz J, Donaldson JG, Schweizer A, Berger EG, Hauri HP, Yuan LC, Klausner RD (1990) Microtubule-dependent retrograde transport of proteins into the ER in the presence of brefeldin A suggests an ER recycling pathway. Cell 60:821-836.

Lohof AM, Ip NY, Poo M-m (1993) Potentiation of developing neuromuscular synapses by the neurotropins NT-3 and BDNF. Nature 363:350-353.

Maniotis AJ, Chen CS, Ingber DE (1997) Demonstration of mechanical connections between integrins, cytoskeletal filaments, and nucleoplasm that stabilize nuclear structure. Proc Natl Acad Sci USA 94:849-854.

Martenson C, Stone K, Reedy M, Sheetz M (1993) Fast axonal transport is required for growth cone advance. Nature 366:66-69.

Miller KW, Joshi HC (1996) Tubulin transport in neurons. J Cell Biol 133:1355-1366.

Moritz M, Braunfeld MB, Sedat JW, Alberts B, Agard DA (1995) Microtubule nucleation by gamma-tubulin-containing rings in the centrosome. Nature 378:638-640.

Okabe S, Hirokawa N (1990) Turnover of fluorescently labelled tubulin and actin in the axon. Nature 343:479-482.

Okabe S, Hirokawa N (1992) Differential behavior of photoactivated microtubules in growing axons of mouse and frog neurons. J Cell Biol 117:105-120.

Okabe S, Hirokawa N (1993) Do photobleached fluorescent microtubules move? Re-evaluation of fluorescence laser photobleaching both in vitro and in growing Xenopus axon. J Cell Biol 120:1177-1186.

Popov SV, Poo M-m (1992) Diffusional transport of macromolecules in developing nerve processes. J Neurosci 12:77-85.

Popov SV, Brown A, Poo M-m (1993) Forward plasma membrane flow in growing nerve processes. Science 259:244-246.

Reinch SS, Mitchison TJ, Kirschner MW (1991) Microtubule polymer assembly and transport during axonal elongation. J Cell Biol 115:365-379.

Rodionov VI, Lim S-S, Gelfand VI, Borisy GG (1994) Microtubule dynamics in fish melanophores. J Cell Biol 126:1455-1464.

Sabry J, O'Connor TP, Kirschner MW (1995) Axonal transport of tubulin in Ti1 pioneer neurons in situ. Neuron 14:1247-1256.

Slaughter T, Wang J, Black MM (1997) Microtubule transport from the cell body into the axons of growing neurons. J Neurosci 17:5807-5819.

Spitzer NC, Lamborghini JE (1976) The development of the action potential mechanisms of amphibian neurons isolated in culture. Proc Natl Acad Sci USA 73:1641-1645.

Stoop R, Poo M-m (1995) Potentiation of transmitter release by ciliary neurotrophic factor requires somatic signaling. Science 267:695-699.

Takeda S, Funakoshi T, Hirokawa N (1995) Tubulin dynamics in neuronal axons of living zebrafish embryos. Neuron 14:1257-1264.

Tanaka E, Ho T, Kirschner MW (1995) The role of microtubule dynamics in growth cone motility and axonal growth. J Cell Biol 128:139-155.

Wang T, Xie K, Lu B (1995) Neurotrophins promote maturation of developing neuromuscular synapses. J Neurosci 15:4796-4805.

Zheng J, Lamoureux P, Santiago V, Dennerll T, Buxbaum RE, Heidemann SR (1991) Tensile regulation of axonal elongation and initiation. J Neurosci 11:1117-1125.

Zheng J, Buxbaum RE, Heidemann SR (1993) Investigation of microtubule assembly and organization accompanying tension-induced neurite initiation. J Cell Sci 104:1239-1250.

Zheng Y, Wong ML, Alberts B, Mitchison T (1995) Nucleation of microtubule assembly by a gamma-tubulin-containing ring complex. Nature 378:578-583. 